\title{
Major Steps in the Discovery of Adiabatic Shear Bands
}

\author{
BRADLEY DODD, STEPHEN M. WALLEY, RONG YANG, \\ and VITALI F. NESTERENKO
}

\begin{abstract}
The standard story of the discovery of adiabatic shear bands is that it began with the American researchers Zener and Hollomon's famous 1944 paper where the phenomenon was first reported and named. However, a recent discovery by one of us (SMW) in the Cambridge University Library has shown that the phenomenon was discovered and described by a Russian researcher, V.P. Kravz-Tarnavskii, in 1928. A follow-up paper was published by two of his colleagues in 1935. Translations of the 1928 and 1935 papers may be found at http://arxiv.org/abs/1410.1353.
\end{abstract}

DOI: $10.1007 / \mathrm{s} 11661-015-2739-1$

(C) The Minerals, Metals \& Materials Society and ASM International 2015

\section{INTRODUCTION}

WHEN one of us (BD) co-authored with Prof. Yilong Bai a book on adiabatic shear bands (ASBs) in $1992,{ }^{[1]}$ the standard story of the discovery of ASBs was believed to have started with the American researchers Zener and Hollomon's famous 1944 paper in the Journal of Applied Physics ${ }^{[2]}$ where the phenomenon was first reported and named. The story then moved back to France in the 1870 s to Henri Tresca's reports on heat crosses seen in the forging of platinum alloys. ${ }^{[3,4]}$ Tresca's study was repeated in England by Massey in 1921 using stee ${ }^{[5]}$ but the first photograph of the heat cross phenomenon was taken by Johnson and coworkers in $1964,{ }^{[6]}$ again using steel.

A close reading of Zener and Hollomon's 1944 paper reveals that although they used the three words 'adiabatic,' 'shear,' and 'band' in their paper, they did not put the three words together to form a single phrase. However, there can be no doubt that they observed ASBs, as can be seen from Figure 1, reproduced from their paper. It is a moot point whether the heat crosses reported earlier by Tresca and Massey should be called 'adiabatic shear bands' as the shear localization they saw is much more diffuse than that shown in Figure 1, as can be seen from the photograph of the phenomenon taken later by Johnson and co-workers (Figure 2).

BRADLEY DODD, formerly with the Institute of Shock Physics, Imperial College, London SW7 2BW, UK, is now Retired. STEPHEN M. WALLEY, formerly Research Associate with the SMF Fracture and Shock Physics Group, Cavendish Laboratory, Cambridge, CB3 0HE, UK, is now Retired. Contact e-mail: smw14@cam.ac.uk RONG YANG, Associate Researcher, is with The State Key Laboratory of Nonlinear Mechanics, Institute of Mechanics, Chinese Academy of Sciences, Beijing 100190, P.R. China. VITALI F. NESTERENKO, Professor, is with the Department of Mechanical and Aerospace Engineering, Jacobs School of Engineering, University of California, San Diego, La Jolla, CA 92093-0403.

Manuscript submitted December 3, 2014.

Article published online January 14, 2015

\section{THE OVERLOOKED RUSSIAN CONTRIBUTION}

So the story rested, and indeed was repeated by one of us in a review paper published in 2007. ${ }^{[7]}$ However, in August 2013, one of us (SMW) found a paper in the Cambridge University Library by Davidenkov and Mirolubov (of the Leningrad Institute of Physics and Technology) entitled: 'A special kind of upset deformation of steel: The KravzTarnavskii effect. ${ }^{[8]}$ This paper is dated 1935 and was published in German in a major Soviet Journal aimed at non-Russian speakers (Technical Physics of the USSR). The editor of this journal was A. Joffe, the father of Soviet physics, and the editorial board included many other major Russian physicists of that time.

Tantalizingly, the 1935 paper is sub-titled 'The KravzTarnavskii Effect' and as you will see from the figures reproduced from this paper (Figures 3, 4, 5), the 'K-T Effect' is in fact adiabatic shear banding. The authors of the 1935 paper refer to a date of 1928 for the first publication of the phenomenon by Kravz-Tarnavskii in Zeitschrift der Russischen Metallurgischen Gesellschaft ${ }^{[9]}$

\section{HUNTING THE JOURNAL}

We were then stuck because there was no record of a journal bearing that name in any library in the United Kingdom or the United States. With hindsight, our assumption that the journal had a German title was wrong (it has a Russian name and was published in Russian). What about Russia itself? How could we perform a search of Russian libraries? Had all copies of the journal printed in 1928 been destroyed during the twentieth century?

Our initial attempts to find the journal containing Kravz-Tarnavskii's paper in Russian libraries by asking for help from Russian experts in this research area were unsuccessful. Moreover, even in the Russian scientific literature, we found only a few examples where references to Kravz-Tarnavskii's and Davidenkov and Mirolubov's papers were made. This is very unusual especially because the phenomenon of shear localization 


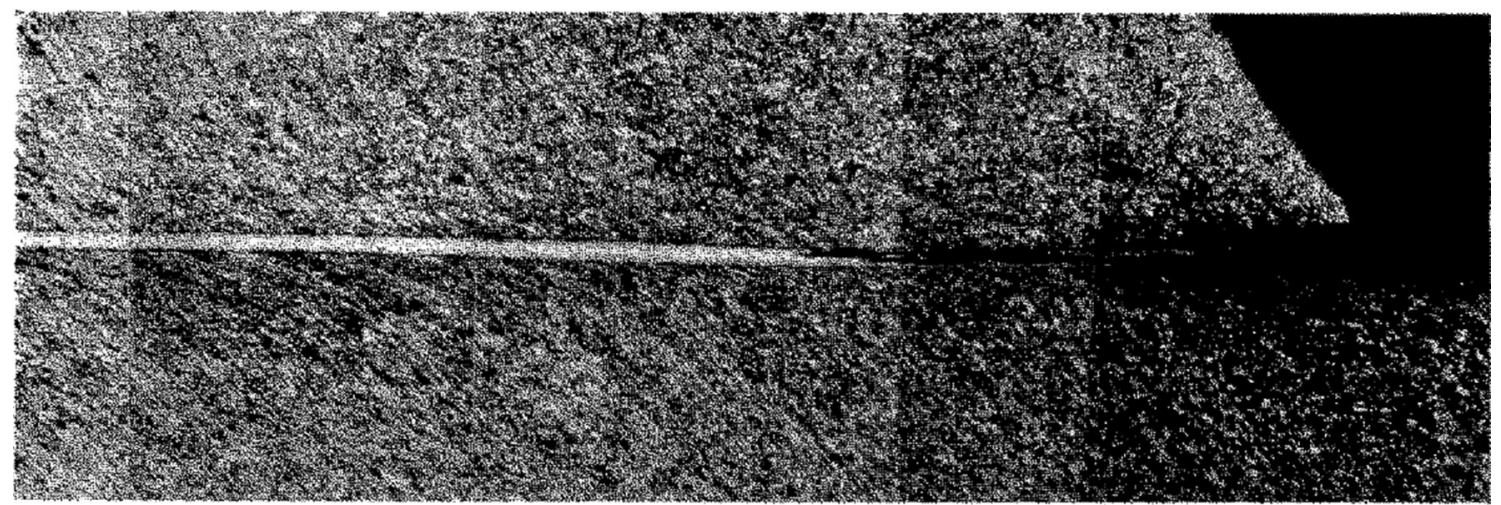

Fig. 1-ASB formed by the dynamic punching of a steel plate using a drop-weight machine. From Zener and Hollomon 1944. ${ }^{[2]}$

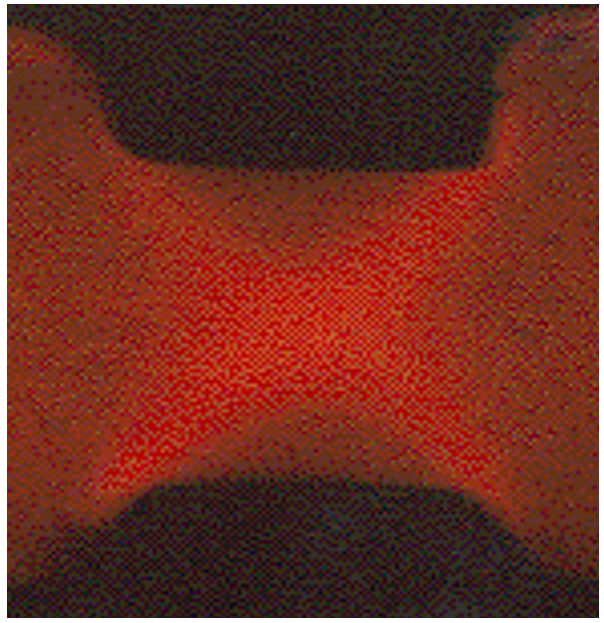

Fig. 2-Photograph of the heat cross in mild steel produced by the punch forging of mild steel. This study was performed by Johnson and co-workers in the same forging shop as Massey used in 1921. From Johnson et al. $1964{ }^{[6]}$

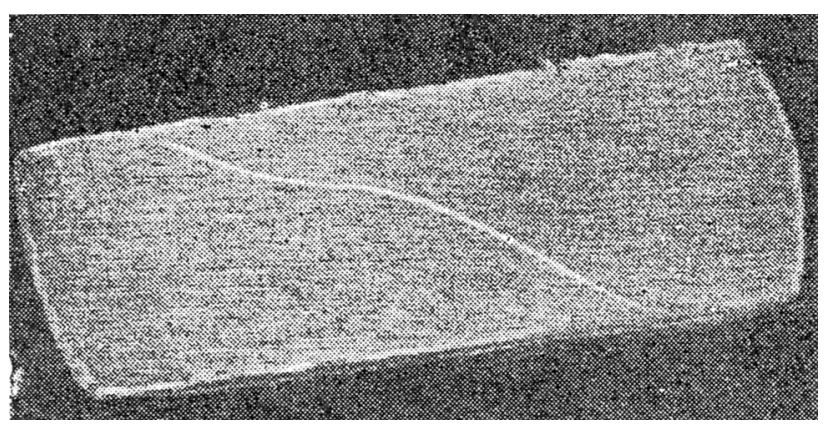

Fig. 3- Shape of band on an etched section of a steel specimen impacted by a $50-\mathrm{kg}$ weight dropped from a height of $2.55 \mathrm{~m}$. From Davidenkov and Mirolubov 1935. ${ }^{[8]}$

first reported in Kravz-Tarnavskii's 1928 paper was later named after him in 1935 in a major Soviet scientific journal. Moreover, the giving of his name to the phenomenon (which is not very often done in the scientific literature and in general only very reluctantly) occurred in a paper co-authored by Davidenkov who later became a Fellow of the Ukrainian Academy of Sciences. He published many papers in the international

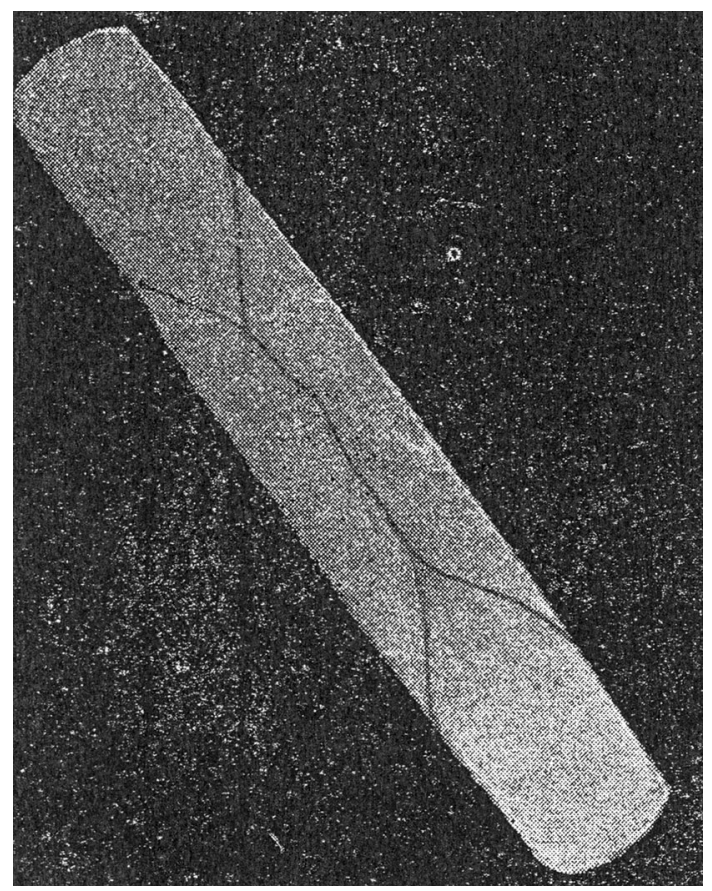

Fig. 4-Form of the band with some branches. From Davidenkov and Mirolubov 1935. ${ }^{[8]}$

literature on metallurgy and he also wrote a book on the dynamic mechanical behavior of metals. ${ }^{[10]}$ But the sad fact is that the two papers by Kravz-Tarnavskii and Davidenkov and Mirolubov, reporting a new phenomenon and its extensive study, were practically forgotten in the scientific literature. This was probably due to the fact that Kravz-Tarnavskii's 1928 paper was published in a Russian journal which would have had limited circulation due to the lack of paper in Russia at that time. Also Davidenkov and Mirolubov's 1935 paper was published in German (a relatively common practice in the 1930s) even though most papers in Technical Physics of the USSR were published in English.

However, we were lucky because a friend of one of us (VFN) found the journal in the central Moscow library in March 2014 and was allowed to photograph KravzTarnavskii's 1928 paper! Rong Yang then translated the Russian text into English, with VFN then checking and 


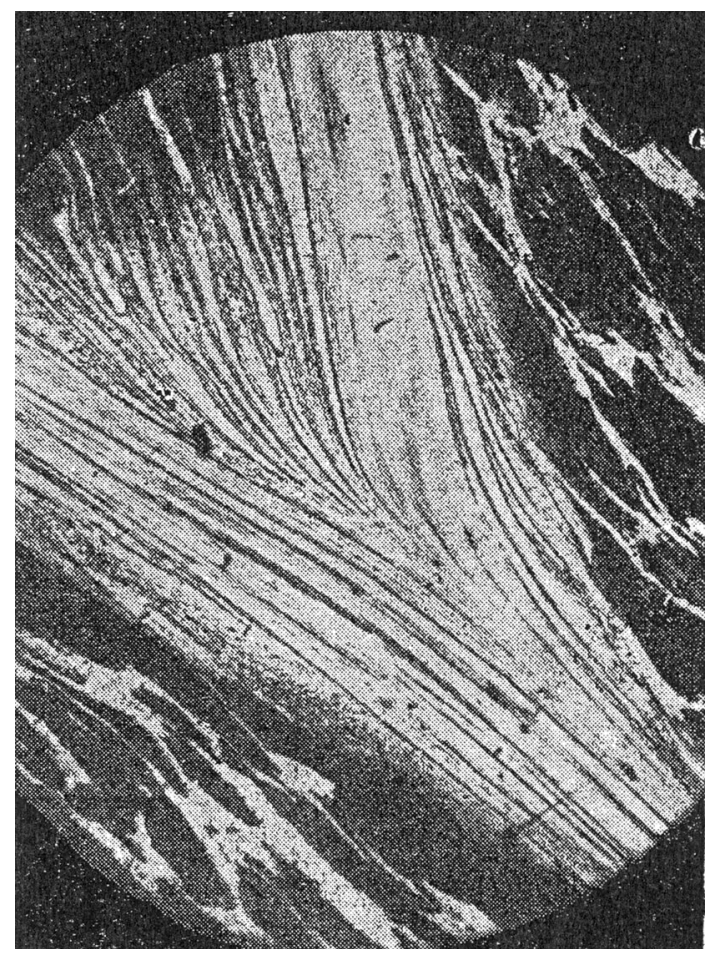

Fig. 5-A band with ferrite penetrating into it. Steel with 0.66 pct C; initial structure: pearlite in ferrite; weight $50 \mathrm{~kg}$, height $4 \mathrm{~m}$. From Davidenkov and Mirolubov 1935. ${ }^{[8]}$

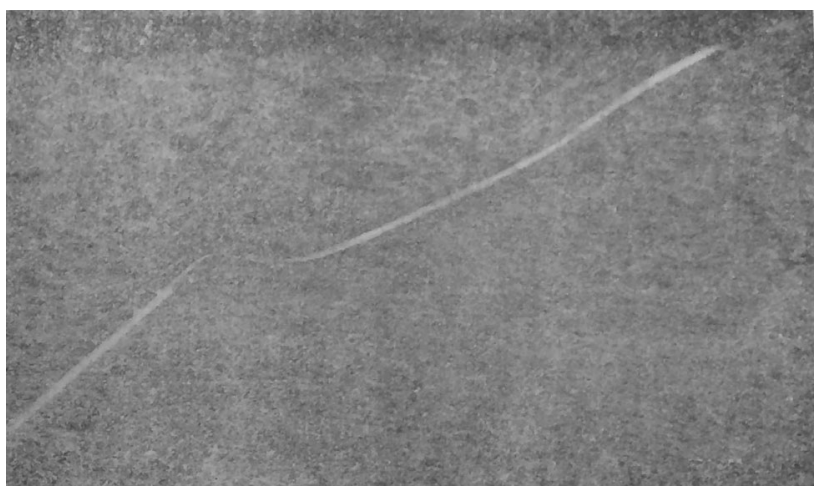

Fig. 6 - Magnification 15 times. From Kravz-Tarnavskii 1928. ${ }^{[9]}$

correcting the translation. The paper is entitled 'A peculiar band discovered in steel.' Rong Yang also performed a literal translation of Davidenkov and Mirolubov's 1935 paper written in German. The translation was then revised by BD and SMW into a more readable form of English and then checked for accuracy by one of our German colleagues, who remarked that the sentence structure was very difficult to understand even for a native speaker of German. We have made these English translations of these 'lost and found' papers available at http://arxiv.org/abs/1410.1353.

A selection of photographs from the 1928 KravzTarnavskii paper is given in Figures 6, 7, 8. These are believed to be the first published photographs of the ASB phenomenon. The figure captions in the original paper simply gave the magnification of the original

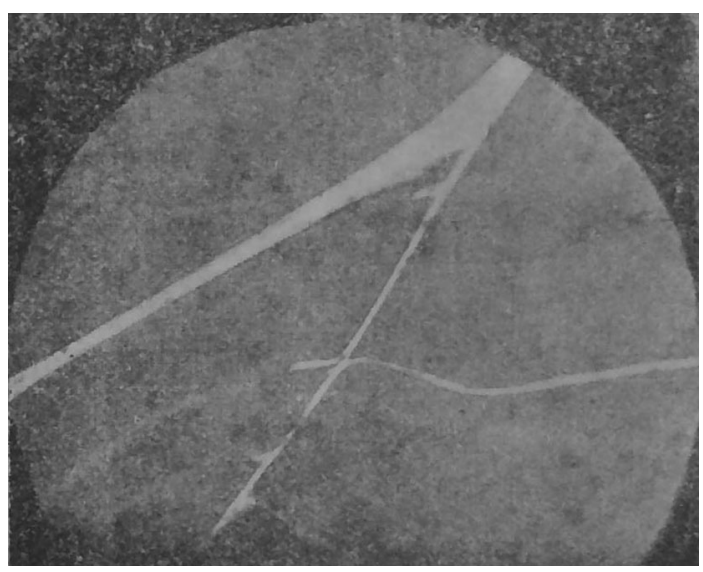

Fig. 7-Magnification 50 times. From Kravz-Tarnavskii 1928. ${ }^{[9]}$

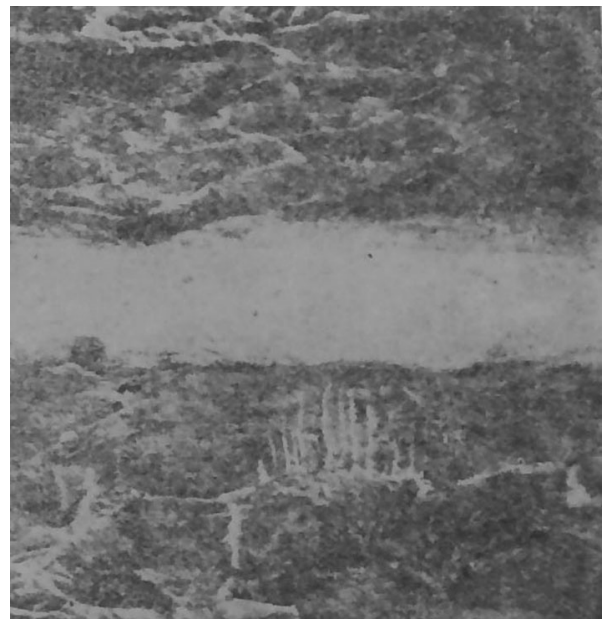

Fig. 8-Magnification 150 times. From Kravz-Tarnavskii 1928. ${ }^{[9]}$

photographs, and this has been respected in their reproduction in this article.

\section{REWRITING THE HISTORY}

These two papers make it clear that the history of the discovery of ASBs should be revised as follows:

1. 1870s: Tresca mentioned that during forging plastic flow can localize into the shape of an $\mathrm{X}$ and that during the dynamic plastic deformation heat was generated ${ }^{[3,4]}$ Massey in England subsequently published a study of these heat crosses in $1921^{[5]}$ but it is clear from the repeat of Massey's experiments carried out by Johnson and co-workers in the $1960 \mathrm{~s}^{[6]}$ that the localization of strain is more diffuse than in true ASBs. The X-shaped region of high temperature can be interpreted as the manifestation of the largest shear strain. This is expected to lie at around 45 deg to the impact direction and under some conditions may be the precursor to the formation of true ASBs. 
2. 1928: Kravz-Tarnavskii first described true ASBs generated in specimens of various types of steel by drop-weight impact at $10 \mathrm{~m} / \mathrm{s} .{ }^{[9]} \mathrm{He}$ made the following important statements based on his observations:

a. quasistatic deformation never resulted in shear localization;

b. dynamic deformation produced by impact was a necessary condition for observation of this 'peculiar band' (shear localization zone) in different steel types with carbon content less than $0.8 \mathrm{pct}$ and other special (though unspecified) steels;

c. occurrence of this 'peculiar band' resulted in low-energy absorption by the rest of the specimen, the energy of deformation being mostly absorbed by the 'peculiar band';

d. the 'peculiar band' had a microstructure very different to the initial microstructure of the steel, in some cases being practically non-etchable, and with a high scratch hardness;

e. he suggested that mass transfer (expulsion of ferrite from the shear localized zone) and subsequent refinement of the microstructure by fragmentation of brittle components was responsible for its unusual properties. We would like to comment at this point that it is unclear how to accomplish mass transfer from a localized shear band which would involve normally slow diffusion processes unless the diffusion is greatly accelerated in the strongly sheared material and also in material nearby. But it should be noted that very finely fragmented microstructure was observed some years later inside localized shear bands in brittle granular materials. ${ }^{[1]}$

3. 1934: Taylor and Quinney found experimentally that the amount of heat produced by plastic deformation was about 90 pet for most metals. ${ }^{[12]}$

4. 1935: Independently Davidenkov and Mirolubov performed extensive experiments on a variety of steels with various microstructures and under different conditions of impact (energy and velocity) in collaboration with Kravz-Tarnavskii (even using some of his samples). ${ }^{[8]}$ They obtained many ASBs under certain impact conditions. They measured the thermal energy dissipated in the deformed samples with and without shear bands and observed no difference between corresponding values. They proposed two different mechanisms of shear localization according to their observation of the dynamic behavior of steels processed under various conditions to produce different initial microstructures in order to explore the role of microstructure on the formation of localized shear:

a. Plastic deformation first occurs in the plane of maximum shear stress accompanied by local heating. Heating lowers the yield stress, so hardening is followed by weakening and further localized deformation continues along the same plane rather than in other regions;

b. Microfracture along the plane of maximum shear stress forms an inclined crack, breaking the specimen into two parts. Subsequent sliding in the plane of the crack results in localized heating so that the crack welds itself together. This is followed by quenching of the heated metal layer which undergoes an austenitic transformation as it is quenched. Based on temperature estimates, they suggested that localized heating followed by rapid quenching due to the surrounding cold bulk metal may result in a martensitic transformation inside the shear band. The quenched microstructure in the localized shear band is different to the normal martensite formed by quenching. They suggested that the reason why the characteristic needle-like martensite microstructure was not formed was due to the high pressure and high rate of transformation. They did not confirm the process of expulsion of ferrite from localized shear zones as suggested in Kravz-Tarnavskii's earlier paper. ${ }^{[9]}$ The studies by Davidenkov and Mirolubov are quite sophisticated, and even show side-pressings as reported much later in the 1980 s by an American team. So at last we now know of the 'lost work' carried out in Russia on the ASB phenomenon.

5. 1944: Zener and Hollomon published a famous paper in the Journal of Applied Physics ${ }^{[2]}$ containing photographs of ASBs generated under different loading conditions to the Russian studies. Zener and Hollomon's method consisted of dropping a weight upon a punch in contact with a plate (this may be called 'forced' shear localization). They suggested a mechanism of shear localization similar to the one first proposed by Davidenkov and Mirolubov emphasizing that "a negative stress-strain curve implies an intrinsic instability of the material where deformation cannot be homogeneous; for a region which, by chance, suffers more deformation than the surrounding region and becomes weakened thereby, will continue to deform while the surrounding region undergoes no further strain." They also suggested that the white band of metal joining the punch and plate is martensite resulting from rapid quenching of material inside highly sheared material (shear strain of nearly 100).

\section{NOTE ON TRANSLATION POLICY}

We have used the English convention for transliterating Russian names, rather than the German one. Thus, 'KravzTarnavskii' rather than 'Krawz-Tarnawskij' (which is how his name appears in the sub-title of the 1935 paper, written by Davidenkov and Mirolubov in German).

\section{ACKNOWLEDGMENTS}

The Institute of Shock Physics acknowledges the support of the Atomic Weapons Establishment, Aldermaston, UK and Imperial College, London. 


\section{REFERENCES}

1. Y.L. Bai and B. Dodd: Adiabatic Shear Localization: Occurrence, Theories and Applications, Pergamon, 1992.

2. C. Zener and J.H. Hollomon: J. Appl. Phys., 1944, vol. 15, pp. 22 32.

3. H. Tresca: Proc. Inst. Mech. Eng., 1878, vol. 29, pp. 301-45.

4. H. Tresca: Ann. Conservatoire Arts Métiers, 1879, vol. 4, pp. 133 50

5. H.F. Massey: Trans. Manchester Assoc. Eng., 1921, pp. 21-66.

6. W. Johnson, G.L. Baraya, and R.A.C. Slater: Int. J. Mech. Sci., 1964, vol. 6, pp. 409-14.
7. S.M. Walley: Metall. Mater. Trans. A, 2007, vol. 38A, pp. 262954.

8. N. Davidenkov and I. Mirolubov: Tech. Phys. USSR, 1935, vol. 2, pp. $281-98$

9. V.P. Kravz-Tarnavskii: J. Russ.Metall. Soc., 1928, issue 3, pp. 162-67.

10. N.N. Davidenkov: Dynamic Testing of Materials (in Russian), ONTI, Leningrad, 1936.

11. C.J. Shih, M.A. Meyers, and V.F. Nesterenko: Acta Mater., 1998, vol. 46, pp. 4037-65.

12. G.I. Taylor and H. Quinney: Proc. R. Soc. Lond. A, 1934, vol. 143 , pp. $307-26$. 